\title{
Milk fat globule-EGF factor 8/lactadherin plays a crucial role in maintenance and repair of murine intestinal epithelium
}

\author{
Heng-Fu Bu, ${ }^{1}$ Xiu-Li Zuo, ${ }^{1,2}$ Xiao Wang, ${ }^{1}$ Michael A. Ensslin, ${ }^{3}$ Vjola Koti, ${ }^{1}$ Wei Hsueh, ${ }^{4}$ \\ Adam S. Raymond, ${ }^{3}$ Barry D. Shur, ${ }^{3}$ and Xiao-Di Tan ${ }^{1}$ \\ ${ }^{1}$ Molecular and Cellular Pathobiology Program and Center for Digestive Disease and Immunobiology, Children's Memorial Research Center, \\ Northwestern University Feinberg School of Medicine, Chicago, Illinois, USA. ${ }^{2}$ Department of Gastroenterology, Qilu Hospital, \\ Medical College of Shandong University, Jinan, Shandong, People's Republic of China. ${ }^{3}$ Department of Cell Biology, Emory University School of Medicine, \\ Atlanta, Georgia, USA. ${ }^{4}$ Department of Pathology, Northwestern University Feinberg School of Medicine, Chicago, Illinois, USA.
}

\begin{abstract}
Milk fat globule-EGF factor 8 (MFG-E8)/lactadherin participates in several cell surface-mediated regulatory events. Although its mRNA is present in the gut, the physiological roles of MFG-E8 in the intestinal mucosa have not been explored. Here we show that MFG-E8 was expressed in intestinal lamina propria macrophages from mice. Using a wound-healing assay, MFG-E8 was shown to promote the migration of intestinal epithelial cells through a PKC $\varepsilon$-dependent mechanism. MFG-E8 bound to phosphatidylserine and triggered reorientation of the actin cytoskeleton in intestinal epithelial cells at the wound edge. Depleting MFG-E8 in mice by administration of anti-MFG-E8 antibody or targeted deletion of the MFG-E8 gene resulted in a slowing of enterocyte migration along the crypt-villus axis and focal mucosal injury. Moreover, in septic mice, intestinal MFG-E8 expression was downregulated, which correlated with intestinal injury, interrupted enterocyte migration, and impaired restitution. Treatment with recombinant MFG-E8 restored enterocyte migration, whereas deletion of MFG-E8 impeded mucosal healing in mice with sepsis. These results suggest that a decrease in intestinal MFG-E8 impairs intestinal mucosal repair in sepsis. Together, our data indicate that MFG-E8 plays an important role in the maintenance of intestinal epithelial homeostasis and the promotion of mucosal healing and suggest that recombinant MFG-E8 may be beneficial for the treatment of bowel injuries.
\end{abstract}

\section{Introduction}

Intestinal mucosa is covered with an epithelial lining that turns over rapidly and continuously throughout life. Cell proliferation, differentiation, and migration are crucial events required for the maintenance of an intact epithelial layer. The stem cells in the crypts of Lieberkühn give rise to progenitor cells followed by transient amplifications through the collaborative action of the Wnt and Notch signaling pathways (reviewed in ref. 1). In physiological state, many progenies of intestinal stem cells differentiate into absorptive enterocytes or secretory cells (including goblet cells and enteroendocrine cells) through multiple binary cell fate decisions regulated by Hes1 and Math1 (2). Differentiation into absorptive enterocytes is associated with loss of Wnt signaling, whereas the acquisition of goblet cell phenotype by progenitor cells is related to loss of Notch signaling. This takes place when cells move upward from the crypt to the tip of villi (1), where they are exfoliated into the lumen of the intestine (3). Concomitantly, some progenies are positioned into the crypt bottom and mature to Paneth cells through activation of a Wnt/TCF4 signal pathway (4).

Intestinal injury often accompanies severe systemic inflammatory response and traumatic stress. The damaged intestinal epithelial lining undertakes restitution, following which the injured epithelium is repaired by cells migrated from the crypts. Restitu-

Nonstandard abbreviations used: CLP, cecal ligation and puncture; ITF, intestinal trefoil factor; MFG-E8, milk fat globule-EGF factor 8.

Conflict of interest: The authors have declared that no conflict of interest exists. Citation for this article: J. Clin. Invest. 117:3673-3683 (2007). doi:10.1172/JCI31841. tion is known to be enhanced by various intestinal peptides and cytokines (5). However, the factors or driving forces required for the upward migration of enterocytes from the crypts of the small intestine are largely unknown.

Milk fat globule-EGF factor 8 (MFG-E8)/lactadherin is a glycoprotein originally found in milk and mammary epithelial cells (6). It is one of the major protein components associated with milk fat globule membrane. Yolken et al. showed that MFG-E8 is an important milk mucin-associated defense component that inhibits enteric pathogen binding and infectivity (7). Recently, MFG-E8 has also been found to be expressed in macrophages, dendritic cells, epididymal cells, and sperm (8-10). MFG-E8 is shown to bind several cell surface molecules including phosphatidylserine, integrins $\alpha v \beta 3$ and $\alpha v \beta 5$, and components of the egg coat, or zona pellucida (8-12). Previous investigations demonstrated that endogenous MFG-E8 promotes RGD-dependent cell adhesion via integrins (11), mediates the removal of apoptotic cells through binding to phosphatidylserine (9), modulates VEGF-dependent neovascularization via the induction of integrin-dependent Akt phosphorylation in endothelial cells (13), and facilitates the sperm-egg interaction by binding to zona pellucida (10). Thus MFG-E8 may be crucial for cell surfacemediated regulatory events. MFG-E8 mRNA has previously been shown to be present in the gut (14). However, it is still largely unknown how endogenous MFG-E8 functions in the intestine. In this study, we localized MFG-E8 in the murine small intestine. Furthermore, we investigated the role of MFG-E8 in intestinal epithelial cell migration and mucosal repair, using in vitro and in vivo models. 

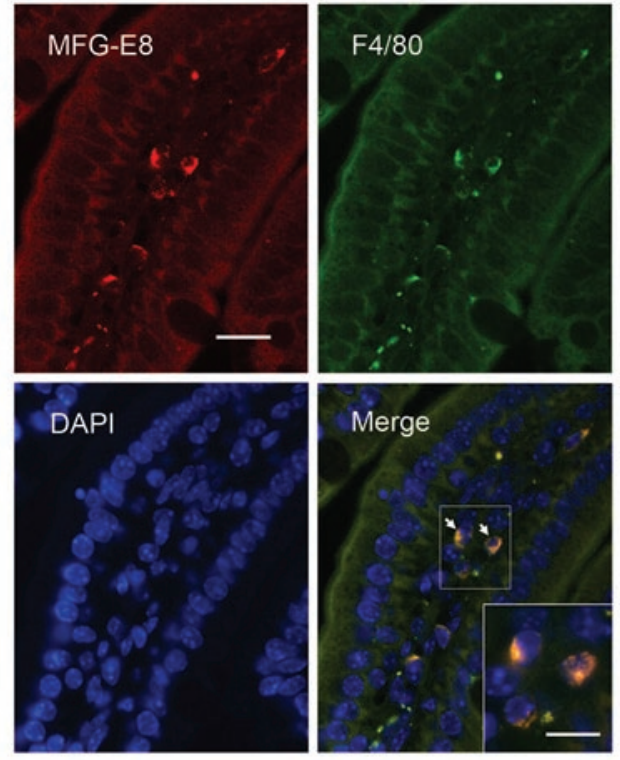

\section{Results}

MFG-E8 is constitutively expressed in macrophages in the lamina propria of the murine small intestine. Small intestinal tissue was dually stained for MFG-E8 and F4/80 (a specific protein marker for macrophages). As shown in Figure 1, the cells strongly expressing MFG-E8 were also positive for F4/80. The control stain (nonimmune IgG used instead of anti-MFG-E8 and anti-F4/80) was negative (Supplemental Figure 1; supplemental material available online with this article; doi:10.1172/JCI31841DS1), indicating the specificity of the staining for MFG-E8 and F4/80. Thus MFG-E8 is specifically expressed by lamina propria macrophages of murine small intestine.

MFG-E8 promotes the migration of intestinal epithelial cells. First, we assessed whether MFG-E8 stimulates intestinal epithelial cell migration, utilizing a classic in vitro wound-healing model. As shown in Figure 2, A and B, serum starved IEC-18 cells spontaneously migrated within 5 hours after wounding. Treatment of the cells with MFG-E8 (5 nM) markedly enhanced the cell migration into the denuded area. The enhancement of IEC-18 cell migration was dose dependent (Figure 2C). MFG-E8 treatment did not interfere with IEC-18 cell proliferation (Supplemental Figure 2) or protect the cells against reactive oxygen species-induced injury (Supplemental Figure 3). Thus the effect of MFG-E8 on cell migration is not due to cell proliferation or cytoprotection.

PKCE mediates MFG-E8 effect on intestinal epithelial cell migration in vitro. In a preliminary experiment, we found that MFG-E8-induced IEC-18 cell migration was reduced by broad-range PKC inhibitors (data not shown). Thus we next identified the PKC isoforms that contribute to MFG-E8 action. Because the role of the PKC pathway in IEC-18 cell movement is not clear, we first performed

\section{Figure 2}

Role of MFG-E8 in intestinal epithelial cell migration in vitro. IEC-18 cells were processed for wound-healing assay as described in Methods. (A) MFG-E8-induced IEC-18 cell migration in vitro. Scale bars: $150 \mu \mathrm{m}$. (B) Treatment with MFG-E8 for 5 hours resulted in a significant increase in cell migration $(n=10)$. (C) MFG-E8 affects cell migration in a dose-dependent fashion $(n=9)$. ${ }^{\star \star} P<0.01$ versus control.

\section{Figure 1}

Localization of MFG-E8 in the murine small intestine. Double immunofluorescent staining of normal mouse small intestine using antibodies against MFG-E8 and F4/80. Arrows in the merge panel indicate mononuclear cells in the lamina propria that are positive for both MFG-E8 and F4/80 shown at higher magnification in the inset. Scale bar: $20 \mu \mathrm{m} ; 12 \mu \mathrm{m}$.

wound-healing assay to ascertain the PKC isoforms required for intrinsic IEC-18 cell migration. We found that the spontaneous IEC-18 cell migration was inhibited by selective inhibitors of PKC $\alpha$ or PKC $\delta$ but not V1-2 peptide, a PKCE translocation inhibitor (Supplemental Figure 4), suggesting that activation of PKC $\alpha$ and PKC $\delta$ is essential for the maintenance of IEC-18 cell motility. Therefore we only assessed whether inhibition of PKC $\varepsilon$ affects the MFG-E8 effect on cell migration. As demonstrated in Figure 3A, MFG-E8-induced IEC-18 cell migration was entirely blocked by V1-2 peptide. To confirm that V1-2 peptide affects MFG-E8 action through targeting PKCE but not other molecules associated with the cell mobility, we investigated the effect of PKC $\varepsilon$ silencing on MFG-E8-induced IEC-18 cell migration using the siRNA strategy described by Li et al. (15). First, we proved that treatment with siPKCE (a siRNA for PKCE) resulted in attenuation of PKCE expression in intestinal epithelial cells (Figure 3B). Furthermore, we found that knockdown of PKC $\varepsilon$ with siPKC 8 blocked MFG-E8 effect on cell migration (Figure 3C). Together these data suggest that MFG-E8 enhances intestinal epithelial migration/restitution via a $\mathrm{PKC} \varepsilon$ signaling pathway.

$M F G-E 8$ selectively binds to phosphatidylserine on the posterior zone of cells at the monolayer wound-edge in vitro and interacts with intestinal epithelial cells in vivo. It has not been elucidated how MFG-E8
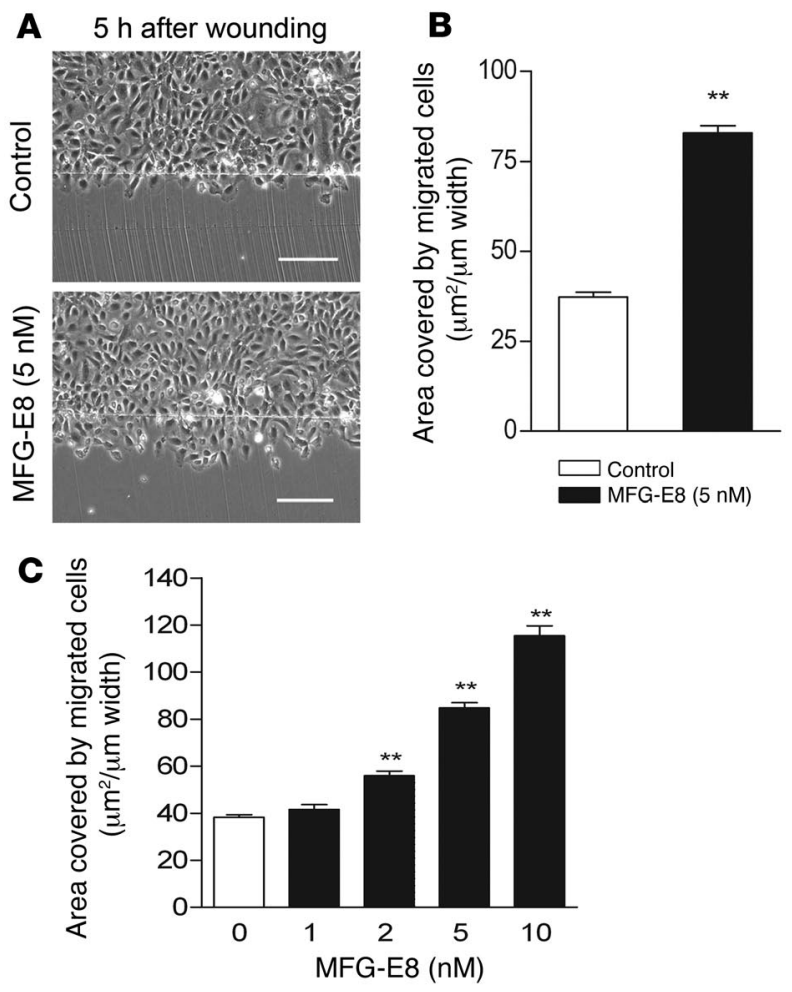


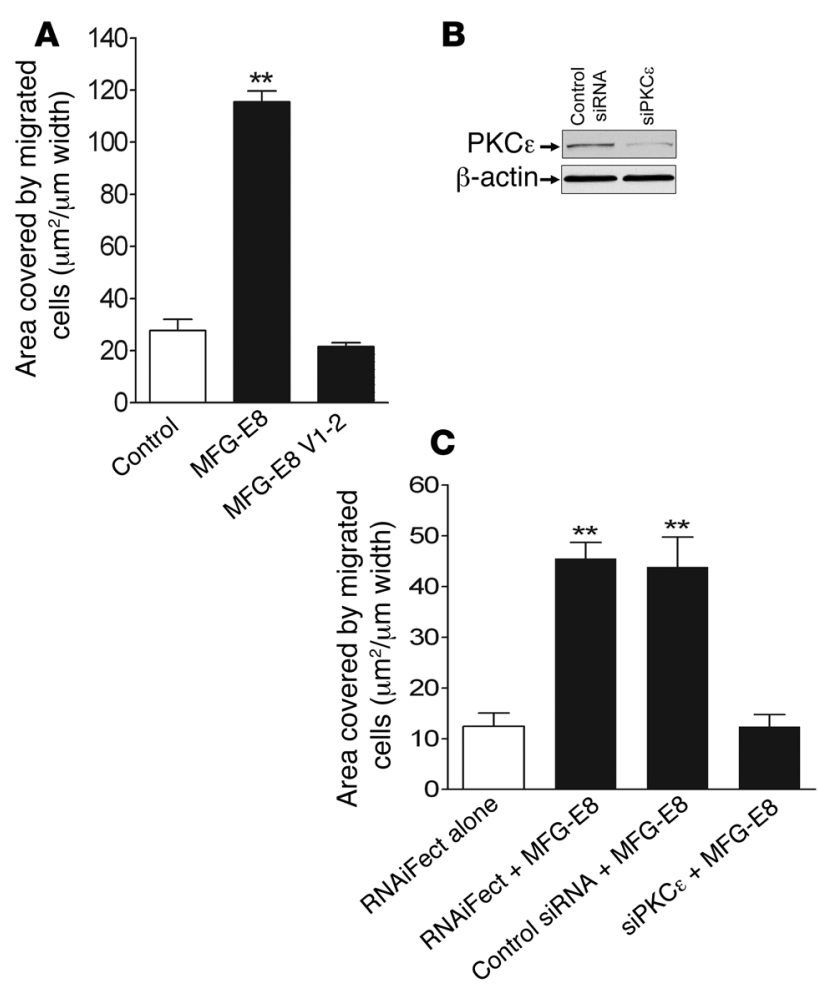

targets intestinal epithelial cells. Because the molecule directly enhances wound healing, we assessed the extent to which MFG-E8 binds to IEC-18 cells using an in vitro model of wounding and restitution. Briefly, we scrape-wounded a confluent IEC-18 cell monolayer. Four hours after spontaneous restitution of the wounded monolayer, the cells were incubated in medium with or without MFG-E8 $(0.5 \mu \mathrm{g} / \mathrm{ml})$ for 15 minutes at room temperature, stained with $\mathrm{mAb}$ to MFG-E8, and examined under a fluorescent microscope. As shown in Figure 4A, anti-MFG-E8 mAb brightly stained MFG-E8-treated IEC-18 cells, which were confined at the wound edge, whereas the control cells stained negative. Interestingly, MFG-E8 binding was noted mostly in the posterior zone (behind the nucleus) of migrating epithelial cells at the wound edge (Figure 4A).

Hanayama et al. demonstrated that MFG-E8 has a high affinity to phosphatidylserine (9). In a pilot study, we found that annexin $\mathrm{V}$, a phosphatidylserine-binding protein, selectively bound to IEC-18 cells at the wound edge (Figure 4B), suggesting transient externalization of phosphatidylserine on the surface of migrating cells. Thus we tested the hypothesis that MFG-E8 binds to transiently exposed phosphatidylserine on cells at the wound edge by competitive blocking of exposed phosphatidylserine with annexin $\mathrm{V}$. As shown in Figure 4C, MFG-E8 binding took place at the posterior zone of cells at the wound edge. Preincubation of cells with a molar excess of annexin $\mathrm{V}(100 \mu \mathrm{g} / \mathrm{ml})$ reduced MFG-E8 binding to the cells, suggesting that MFG-E8 binds phosphatidylserine on the posterior zone of intestinal epithelial cells at the wound edge. Furthermore, we examined whether MFG-E8 and annexin V concurrently bind to the same site in migrating IEC-18 cells by a double-labeling strategy. As shown in Figure 4D, MFG-E8 and annexin $\mathrm{V}$ bound onto intestinal epithelial cells at the wound edge. However, the overlaid image indicates that little MFG-E8 was colocalized with annexin $\mathrm{V}$ on the cell surface, suggesting that

\section{Figure 3}

PKC $\varepsilon$ is required for MFG-E8-induced IEC-18 cell migration. (A) IEC-18 cells were processed for wound-healing assay as described in Methods. Pretreatment with V1-2 $(150 \mu \mathrm{g} / \mathrm{ml}$; a PKC $\varepsilon$ translocation inhibitor peptide) for 90 minutes resulted in attenuation of MFG-E8 (10 nM) effect on cell migration. $n=3 .{ }^{\star \star} P<0.01$ versus control. (B) Western blotting of IEC-18 whole-cell extracts. The cells had been transfected with siPKCE $(1 \mu \mathrm{g} / \mathrm{ml}$; a PKC $\varepsilon$-specific siRNA) or control scrambled siRNA twice with a 24-hour interval. (C) IEC-18 cells were transfected with SiPKC $\varepsilon$ or control siRNA twice with a 24-hour interval, using the RNAiFect reagent. Twenty-four hours after the second siRNA treatment, cells were wounded and allowed to migrate in the presence or absence of MFG-E8 $(10 \mathrm{nM})$ for 7 hours. $n=6 .{ }^{* \star} P<0.01$ versus control.

phosphatidylserine residues occupied by MFG-E8 were not further available to annexin $\mathrm{V}$ or vice versa.

We also examined whether MFG-E8 binds to intestinal epithelial cells in vivo. Septic mice were used because inflammation could accelerate the distribution of MFG-E8 into the intestinal lamina propria due to increased vascular permeability. Briefly, we challenged animals with cecal ligation and puncture (CLP). Twentyfour hours after surgery, animals received a bolus injection of MFG-E8 $(2 \mu \mathrm{g} / \mathrm{g}$, i.v. $)$ and were sacrificed 60 minutes later. Frozen sections of small intestines were stained with anti-MFG-E8 mAb. As shown in Figure 4E, the mAb against MFG-E8 recognized molecules localized in the basal layer of epithelium in mice injected with MFG-E8. In contrast, the tissue was not stained by the isotype control IgG (Figure 4F). The data suggest binding of MFG-E8 by intestinal epithelial cells in vivo.

The role of MFG-E8 in reorganization of F-actin-associated cytoskeleton in migrating intestinal epithelial cells. Intestinal epithelial cell migration involves formation of lamellipodia and lamellae, a complex and dynamic process dependent on the reorganization of F-actin cytoskeleton $(16,17)$. It occurs within 2 hours after wounding in vitro (17). To investigate the mechanism whereby MFG-E8 modulates epithelial cell mobility, we treated serum-starved IEC-18 cells with MFG-E8 (10 nM) for 2 hours, stained the cells with FITC-phalloidin, and examined the F-actin-associated lamellipodia/lamellae structure. We identified 2 types of migrating cells with distinctive actin filament structures at the migrating front: type I (Figure 5A), cells with a large amount of F-actin stress fibers forming lamellum-like protrusions, and type II (Figure 5A), cells with a polarized morphology, a ruffling lamellipodium at the leading edge facing the direction of migration, followed by a flat lamellum and reduced stress fibers. Dually immunostained cells for F-actin and Arp2/3 (to localize actin-associated proteins) revealed colocalization of Arp $2 / 3$ and F-actin in lamellipodia at the leading edge of type II (but not type I) migrating cells (Figure 5B). Quantitative analysis showed that in the controls only $12 \%$ of the cells at the migrating front were type II cells. After MFG-E8 treatment the number of type II cells at the migrating front increased to $75 \%$ (Figure 5C). Together the data suggest that MFG-E8 treatment triggers the reorientation of F-actin and its associated proteins in migrating intestinal epithelial cells, which may facilitate the cell movement.

MFG-E8 is critical for the maintenance of intestinal epithelial homeostasis in vivo. To assess the physiological role of MFG-E8 in intestinal epithelial cell migration in vivo, we treated mice with hamster anti-murine MFG-E8 mAb (2 mg/kg, i.p., $n=5)$. The specificity of the antibody was confirmed by western blot. The control ani- 

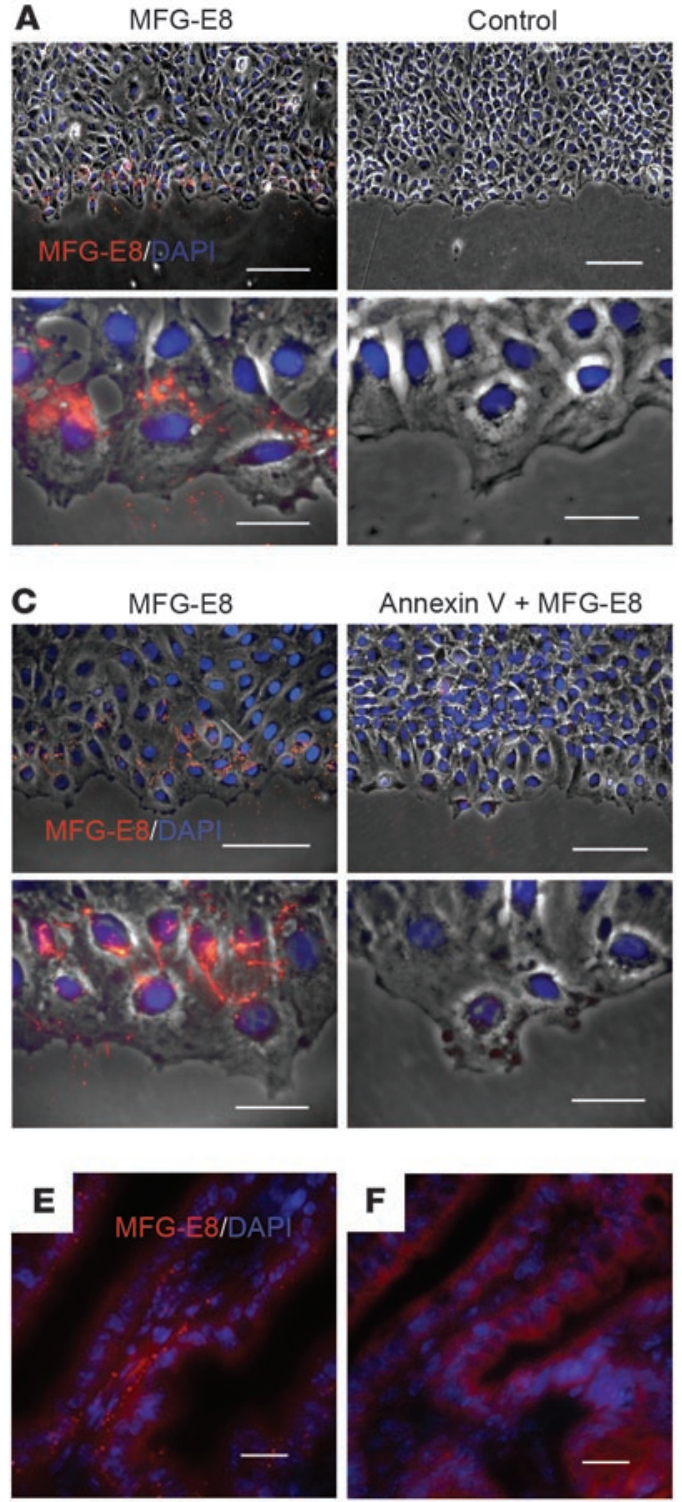

B
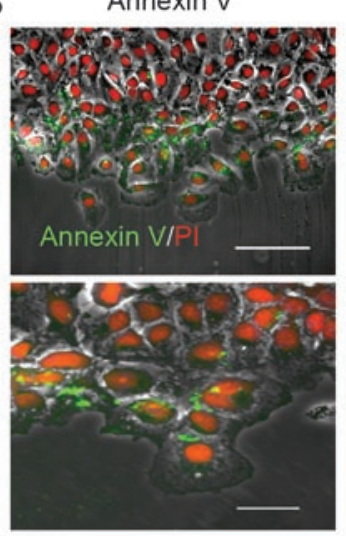

D
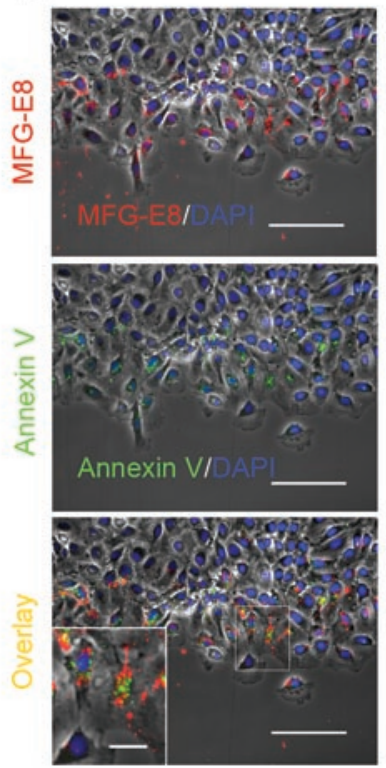

Figure 4

MFG-E8 binds to phosphatidylserine on IEC18 cells at the monolayer wound-edge in vitro and binds to intestinal epithelial cells in vivo. (A) Wounded IEC-18 monolayers were cultured for 4 hours in serum-free medium, incubated for $15 \mathrm{~min}$ utes with MFG-E8 (5 nM) or control medium, and immunostained with anti-MFG-E8 antibody. Nuclei were counterstained with DAPI. Scale bars: 150 um (top panels); $30 \mu \mathrm{m}$ (bottom panels). (B) Cells were treated as in A, but FITC-annexin V $(5 \mu \mathrm{l} / 100$ $\mu$ l) was used instead of MFG-E8 and nuclei were counterstained with propidium iodide $(\mathrm{PI})$. Scale bars: $100 \mu \mathrm{m}$ (top panel); $30 \mu \mathrm{m}$ (bottom panel). (C) Wounded IEC-18 monolayers were incubated for 15 minutes in medium (MFG-E8 group) or medium supplemented with a 200 -fold molar excess of annexin V (annexin V + MFG-E8 group) and processed for MFG-E8 binding experiment as described in A. Scale bars: $100 \mu \mathrm{m}$ (top panels); $30 \mu \mathrm{m}$ (bottom panels). (D) Cells were treated as in A, except that a cocktail containing MFG-E8 $(0.5 \mu \mathrm{g} / \mathrm{ml})$ and FITC-annexin $\mathrm{V}(5 \mu \mathrm{l} / 100 \mu \mathrm{l}$ binding buffer) was used. The inset shows a selected area in the overlaid image at higher magnification. Scale bars: $100 \mu \mathrm{m}$; $25 \mu \mathrm{m}$ (inset). (E) MFG-E8 interacts with intestinal epithelial cell in vivo. MFG-E8 (2 $\mu \mathrm{g} / \mathrm{g}$, i.v.) was injected into CLP-challenged mice. Sixty minutes after MFG-E8 administration, frozen sections of the small intestine were prepared and immunostained with anti-MFG-E8 antibody. Scale bar: $30 \mu \mathrm{m}$. (F) Negative control of immunostaining for MFG-E8. Animals were treated as in $\mathbf{E}$, but isotopic control IgG was used instead of anti-MFG-E8 antibody. Scale bar: $30 \mu \mathrm{m}$. mals $(n=5)$ were given hamster IgG isotype control. After overnight antibody administration, we examined enterocyte migration in vivo by a labeling experiment with BrdU. Tracking the movement of BrdU-labeled enterocytes for up to 48 hours revealed that enterocytes spontaneously migrated along the crypt-villus axis in the small intestine in control IgG-treated mice (Figure 6A), whereas the migration of BrdU-labeled cells was delayed in the mice treated with anti-MFG-E8 antibody (Figure 6A). Statistical analysis revealed that neutralization of endogenous MFG-E8 with anti-MFG-E8 $\mathrm{mAb}$ resulted in a significant decrease in cell migration along the crypt-villus axis (Figure 6B). Histological examination of intestinal tissues revealed a mild focal injury at villous tips in animals treated with anti-MFG-E8 antibody (Figure 6C). In addition, we examined enterocyte crypt-to-villus migration in the small intestine of MFG-E8-knockout and wild-type mice using the BrdU-labeling study to rule out possible off-target effects of anti-MFG-E8 antibody. Compared with wild-type animals, MFG-E8-deficient mice showed markedly reduced enterocyte migration (Figure 6D). These mice also showed a mild focal muco- sal injury at villous tips, with a few necrotic epithelial cells; the pathology was similar to that in anti-MFG-E8 antibody-treated wild-type animals (Figure 6E). Taken together, our data suggest that MFG-E8 plays an important role in the maintenance of intestinal epithelial homeostasis in vivo.

Sepsis-triggered intestinal injury is associated with a downregulation of intestinal MFG-E8 and a delayed enterocyte migration along the cryptvillus axis. We further investigated whether intestinal MFG-E8 expression was changed in sepsis, using the CLP model (18). In preliminary experiments, we found that microscopic injury of the small intestine occurred rapidly after sublethal CLP. Focal villous necrosis developed in the ileum 48 hours after CLP, but only villous edema was noted in the jejunum and duodenum (Supplemental Figure 5). Mucosal damage was gradually repaired within the next 48 hours, during resolution of acute systemic inflammatory response. To correlate CLP-induced intestinal injury with MFG-E8 expression, we first confirmed that the number of macrophages was not affected by CLP, as the intestinal F4/80 protein was not changed at 48 or 72 hours after CLP (Supplemental Figure 6). 
A
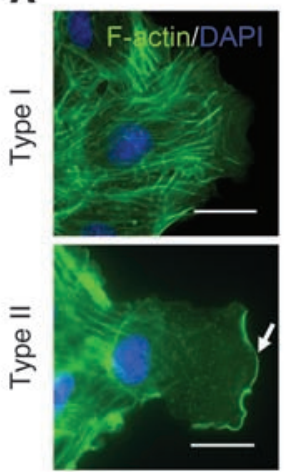

B
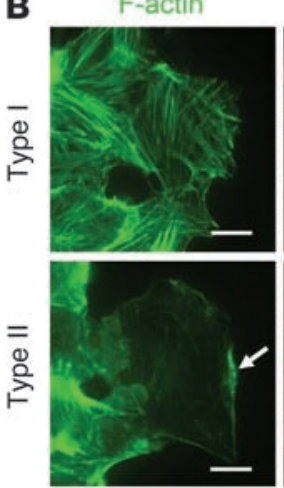

Arp2/3
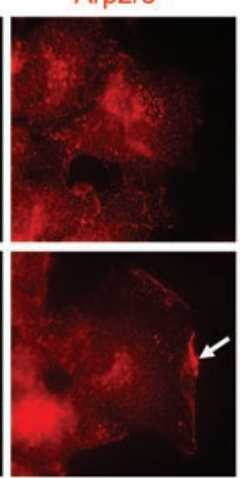

DAPI
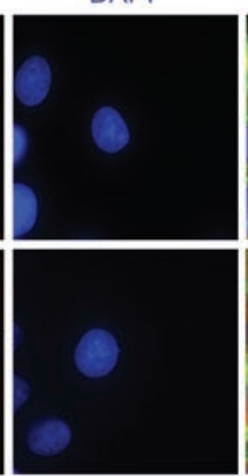

Merge
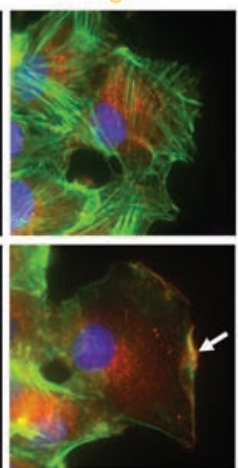

C

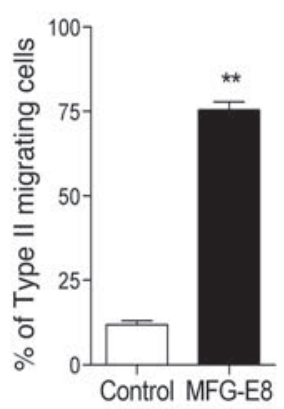

Figure 5

MFG-E8 treatment results in reorganization of F-actin and Arp2/3 in migrating intestinal epithelial cells. (A) Actin filament structures in types I and II migrating cells. Arrow indicates lamellipodia. Scale bars: $15 \mu \mathrm{m}$. (B) Localization of Arp2/3 in types I and II migrating cells at the wound edge. Arrows indicate lamellipodia. Scale bars: $15 \mu \mathrm{m}$. (C) MFG-E8 triggers formation of type II migrating intestinal epithelial cells. Migrating IEC-18 cells were incubated for 2 hours in control medium or medium supplemented with MFG-E8 (10 nM) followed by staining with FITC-conjugated phalloidin. The number of type II migrating cells at wound edge was counted as described in Methods. $n=6 .{ }^{* \star} P<0.01$ versus control.

Then we assessed intestinal MFG-E8 expression in CLP-challenged mice by using western blot analysis. As shown in Figure 7, A and $\mathrm{B}$, sham operation did not affect intestinal MFG-E8 expression. In contrast, intestinal MFG-E8 expression was markedly downregulated within 48 hours after CLP. MFG-E8 expression was partially recovered but remained low at 72 hours after CLP.

We also examined the expression of ITF, a potent restitution and cytoprotective factor secreted by goblet cells (19-21), in animals challenged with CLP. As shown in Figure 7, C and D, CLP induced a transient upregulation of ITF in the small intestine. Together the data suggest that the decrease in intestinal MFG-E8 expression is a particular event during sepsis. The change of intestinal MFG-E8 expression is correlated with the time sequences of developing and resolving intestinal injury in CLP mice.

Given the observed decrease in intestinal MFG-E8 expression after CLP, we investigated whether downregulation of MFG-E8 in sepsis is associated with a change in enterocyte migration along the crypt-villus axis, using a labeling experiment with BrdU. The schedule for surgery, BrdU injection, and sacrificing animals is illustrated in Figure 8A. Briefly, mice were subjected to CLP or sham surgery. Forty-eight hours after the surgery, we administrated BrdU i.p. and pulse-chased enterocyte migration along the crypt-villus axis for up to 48 hours. Areas with intact villi were selected to examine enterocyte migration along the crypt-villus axis in sepsis. As shown in Figure 8B, enterocytes in the crypts were rapidly labeled by BrdU within 2 hours after BrdU injection in both CLP and sham groups. The rate of BrdU incorporation into cells in the CLP group was similar to that in the sham group (Figure 8C), indicating that CLP-triggered sepsis has no effect on enterocyte proliferation. Tracking BrdU-labeled cells for 24 and 48 hours revealed that enterocytes migrated along the crypt-villus axis in the sham group (Figure 8, B and D), and the average distance of BrdU-positive cells from the base of the crypt was approximately $1 / 3$ and $2 / 3$ of the villus height at 24 and 48 hours, respectively, after BrdU exposure (Figure $8 \mathrm{E}$ ). Unlike in sham controls, no migration of labeled cells was evident at 24 hours after BrdU exposure in the CLP group (Figure 8, B and D). The BrdU-labeled cell migration along the crypt-villus axis in CLP mice was significantly delayed compared with the sham control mice at 48 hours after BrdU injection (Figure 8, D and E). Taken together, these findings, in conjunction with the data on MFG-E8 expression, indicate that septic intestinal injury is associated with a decrease in intestinal MFG-E8 protein expression and an inhibition of intestinal epithelial migration along the crypt-villus axis. The change of enterocyte migration rate showed a relationship with the alteration of intestinal MFG-E8 expression in sepsis.

The role of MFG-E8 in mucosal healing. The downregulation of intestinal MFG-E8 in sepsis could cause a decrease in enterocyte migration along the crypt-villus axis. To test this hypothesis, we treated CLP-challenged mice with a recombinant murine MFG-E8. Mice were subjected to CLP followed by treatment with MFG-E8 $(n=7)$ or BSA $(n=7)$ using a protocol described in Figure 9A. Since CLP caused a marked decrease in intestinal MFG-E8 expression (Figure 7, A and B) and delayed enterocyte migration (Figure 8 ) between 48 and 72 hours after the surgical operation, animals that survived 46 hours after CLP were treated with MFG-E8 (2 mg/kg, i.p., MFG-E8 dissolved in PBS containing $50 \mu \mathrm{g} / \mathrm{ml}$ BSA). The control mice were treated with BSA alone 46 hours after CLP. Two hours later, the enterocyte migration was pulse-chased for 24 hours using the BrdU-labeling method. As illustrated in Figure 9B, few BrdU-labeled enterocytes moved out from intestinal crypts in the CLP + BSA group. In contrast, BrdU-labeled enterocytes migrated to about $30 \%$ of villus height from crypts in the CLP + MFG-E8 group. Statistical analysis revealed that MFG-E8 significantly enhanced enterocyte migration along the crypt-villus axis (Figure 9C). The data indicate that treatment with MFG-E8 results in restored intestinal epithelial migration along the cryptvillus axis in CLP-challenged mice.

Although MFG-E8 expression decreased at 2 days after CLP (Figure $7, \mathrm{~A}$ and $\mathrm{B}$ ), the protein was recovered in the late period of CLPinduced systemic inflammatory response, which was most likely followed by the repair of the intestinal epithelium (Supplemental Figure 5). To explore the role of MFG-E8 in mucosal healing, wildtype and MFG-E8-null mice were subjected to sublethal CLP. Histological examination of the small intestine from wild-type animals 4 days after CLP revealed intact mucosa and completely repaired 
A

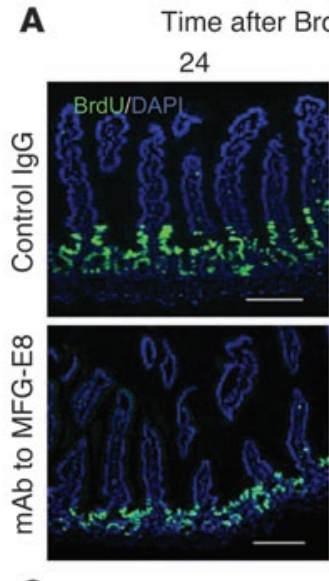

24

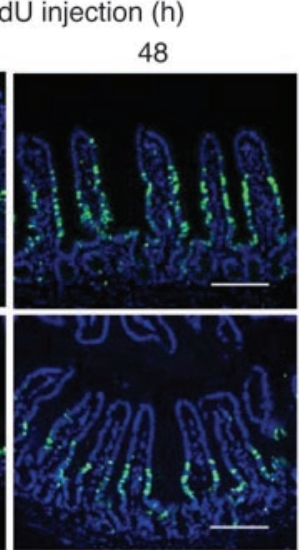

C

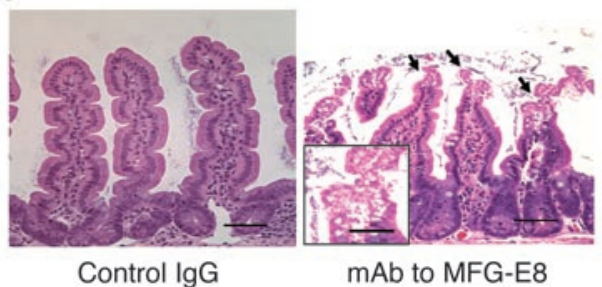

E

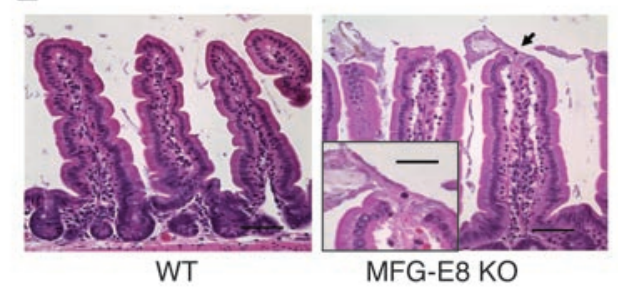

B

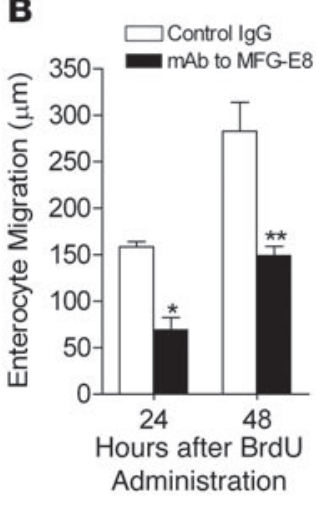

D

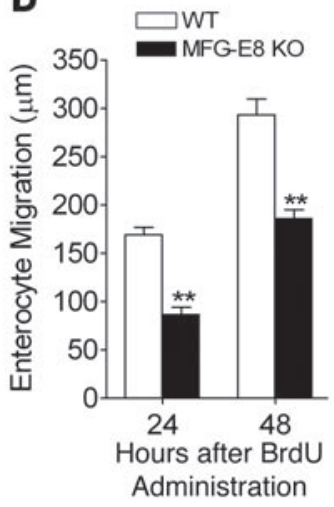

\section{Figure 6}

MFG-E8 depletion slows enterocyte migration along the crypt-villus axis and induces focal villous injury. (A-C) Wild-type mice were treated with $2 \mathrm{mg} / \mathrm{kg}$ (i.p.) of either control IgG or monoclonal anti-MFG-E8 antibody. One day after administration of antibody, BrdU $(50 \mathrm{mg} / \mathrm{kg}$, i.p.) was injected to label enterocytes. The BrdU-labeled enterocyte migration along the crypt-villus axis was traced on tissue sections by immunostaining with anti-BrdU antibody (A) and quantitatively assessed (B) as described in Methods. $n=5$. ${ }^{\star} P<0.05 ;{ }^{\star \star} P<0.01$ versus control. $\mathrm{H} \& \mathrm{E}$ staining $(\mathbf{C})$ shows the histological characteristics of the small intestine from mice treated with control IgG or anti-MFG-E8 mAb for 24 hours. Arrows indicate necrosis of intestinal epithelium, shown at higher magnification in the inset. Scale bars: $100 \mu \mathrm{m} ; 50 \mu \mathrm{m}$ (inset). (D) Pulse-chase experiment revealed a decrease in migration of BrdU-labeled enterocytes from crypts to villus tips in MFG-E8-knockout mice. $n=5 .{ }^{* *} P<0.01$ verses control. (E) H\&E stained wild-type and MFG-E8-deficient intestinal epithelium. Arrow indicates villous tip injury in MFGE8-knockout mice shown at higher magnification in the inset. Scale bars: $100 \mu \mathrm{m} ; 50 \mu \mathrm{m}$ (inset). epithelial layer in all 7 mice (Figure 9D). In contrast, MFG-E8knockout mice $(n=7)$ showed mucosal injury with villous sloughing, which persisted at 4 days after CLP (Figure 9D), indicating that lack of endogenous MFG-E8 impedes mucosal healing.

\section{Discussion}

In the present study, we demonstrate that intestinal MFG-E8 originates from lamina propria macrophages. We provide evidence that MFG-E8 has a potent and dose-dependent in vitro effect on intestinal epithelial cell migration and wound healing. Furthermore, we show that depletion of MFG-E8 with a monoclonal antibody or deletion of the MFG-E8 gene results in a slow-down of intestinal epithelial cell migration along the crypt-villus axis in mice. In addition, we find that the mucosal barrier of the small intestine is disrupted in MFG-E8-deficient mice. Together these findings indicate, for what we believe to be the first time, that MFG-E8 is a crucial factor for enhancing enterocyte migration in vitro as well as in vivo. It helps to maintain mucosal integrity and epithelial homeostasis in the intestinal tract.

It is interesting that MFG-E8 selectively binds onto the surface of intestinal epithelial cells located at the migrating zone of wounded monolayers. MFG-E8 has high affinities to different molecules on the cell membrane that have been shown to mediate MFG-E8 action in a cell type-dependent manner (8-12). With a competitive binding strategy, we demonstrated that MFG-E8 bound to the surface of migrating intestinal epithelial cells by recognizing phos- phatidylserine. This observation is consistent with the previous finding that MFG-E8 is a phosphatidylserine-binding protein (9).

Phosphatidylserine resides largely in the inner leaflet of the plasma membrane in resting cells. Externalization of phosphatidylserine is a traditional hallmark of cells undergoing apoptosis (22). However, it is worth noting that transient exposure of phosphatidylserine also takes place in the cell membrane during several nonapoptotic cellular events such as cell activation and myotube formation $(23,24)$. It has been shown that surface phosphatidylserine exposure occurs at the uropod in AMLP-polarized neutrophils (25). Phosphatidylserine interacts with intracellular proteins such as PKC and Rac1 $(26,27)$, which regulate the actin cytoskeleton and cell mobility. Our observations suggest that phosphatidylserine is transiently externalized when enterocytes are programmed for migration. In view of the signaling cascades uniquely induced by MFG-E8, it remains to be determined whether the binding of MFG-E8 to externalized phosphatidylserine in healing enterocytes promotes the activation of PKC and Rac1, which precedes cell migration.

In addition to phosphatidylserine, MFG-E8 has been shown to bind integrins $\alpha v \beta 3$ and $\alpha v \beta 5$ via its $\operatorname{RGD}$ motif $(8,11,12)$. Integrins play an important role in tissue remodeling and cell migration. Recent studies demonstrate that MFG-E8 induces neovascularization and mammary gland branching morphogenesis through an $\alpha v \beta 3 / 5$ integrin binding-dependent mechanism (13, 28). However, our pilot study indicates that induction of intestinal 

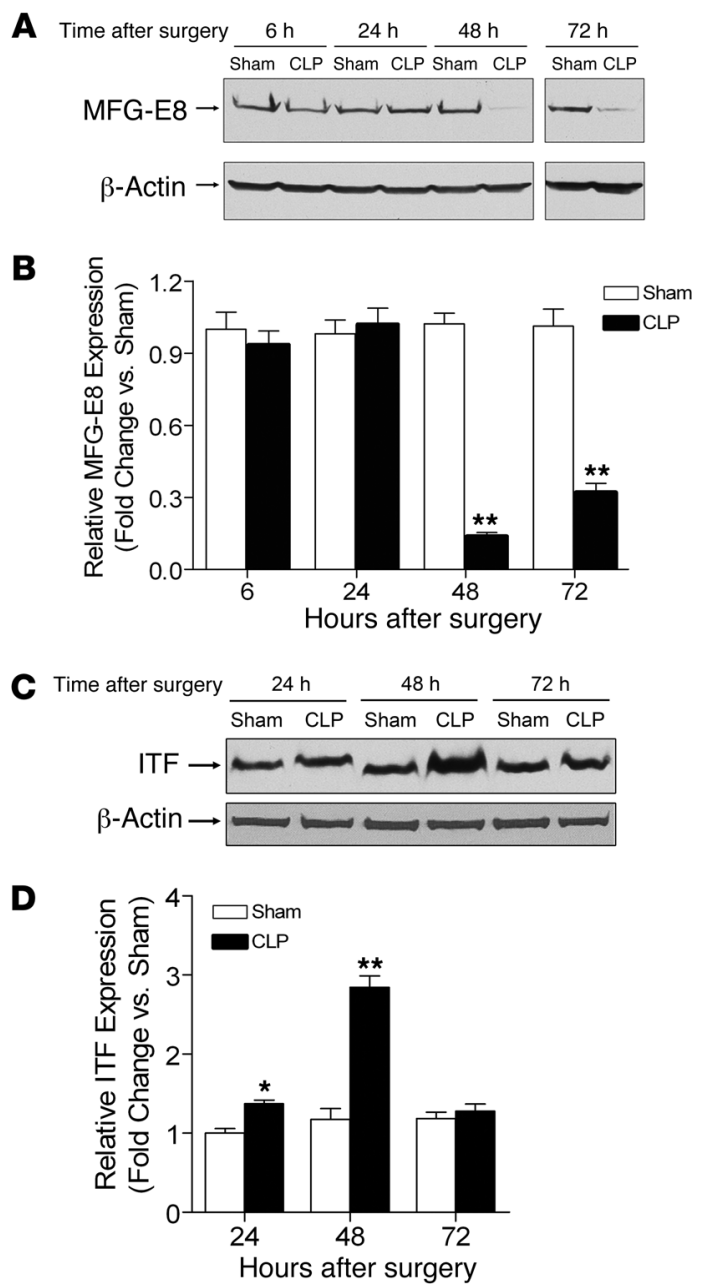

epithelial cell migration in vitro does not require $\alpha v \beta 3 / 5$ integrin ligation (Supplemental Figure 7). Ensslin and Shur (28) reported that MFG-E8 functions as a signaling adhesive molecule, influencing interactions between epithelia and their adjacent cells such as myoepithelial cells. Indeed, subepithelial myofibroblasts have been shown to enhance intestinal epithelial migration (29). Thus we cannot completely rule out the possibility that MFG-E8 enhances enterocyte migration via the RGD motif of MFG-E8 and $\alpha v \beta 3 / 5$ integrin interaction in vivo.

The mechanism of MFG-E8 action on intestinal epithelial cell migration is not clear. Here we demonstrate that MFG-E8 induced intracellular movement of Arp2/3, formation of lamellipodia, and diminishment of F-actin stress fibers in intestinal epithelial cells. Furthermore, our data show that the MFG-E8-triggered rearrangement of actin cytoskeleton was followed by an increase of PKC $\varepsilon$ dependent cell motility. Cell migration is a dynamic process that is associated with cytoskeletal assembly and disassembly. Ridley et al. have shown that the stress fibers in stationary cells are disassembled when epithelial cells receive a migratory stimulus (30) so that they can form broad motile lamellipodia. Stress fibers reappear as they form focal adhesions with the substrate, but they are not found when cells first initiate polarization and formation of the lamellipodia. Collectively, our findings together with others in the literature imply that binding MFG-E8 onto phosphatidylserine on

\section{Figure 7}

The effect of septic challenge on the expression of MFG-E8 and ITF in the small intestine. Mice were subjected to CLP challenge or sham operation as described in Methods. The expression of MFG-E8 and ITF in the small intestine was examined at the indicated intervals after surgery with western blotting. Representative western blots of MFG-E8 (A) and ITF (C) are shown. Densitometric analysis of immunoblot data of MFG-E8 (B), and ITF (D). $n=5$ per group. ${ }^{*} P<0.05$ or ${ }^{* \star} P<0.01$ versus sham group.

intestinal epithelial cells likely triggers rearrangement of F-actinassociated cytoskeletons and changes the mobility of enterocytes in a PKCE-dependent manner. PKCE has been shown to induce phosphorylation of myristoylated alanine-rich $C$ kinase substrate (MARCKS), an important factor for regulating the local F-actin structure (31). Activation of PKC $\varepsilon$ results in remodeling intestinal epithelial F-actin cytoskeleton through a mechanism involving MARCKS (32). Thus we speculated that MFG-E8 may regulate the enterocyte migration via a PKCE to MARCKS to F-actin cascade.

Enterocyte migration along the crypt-villus axis is essential for maintenance of intestinal homeostasis. Although identified for decades, this phenomenon remains unexplained at the molecular level. It has been proposed that the intestinal epithelium is shaped by a series of interactions involving the microbiota, epithelium, and gut-associated lymphoid tissue. Previously, Wong et al. (33) have shown that forced expression of a stable form of $\beta$-catenin in intestinal epithelium results in slowing of cellular migration along the crypt-villus axis. This suggests that Wnt/ $\beta$-catenin signal in intestinal epithelial cells plays a crucial role in controlling the upward movement of enterocytes from crypts to villi. Moreover, Mashimo et al. (34) have demonstrated that enterocyte migration to the mucosal surface requires ITF, a protein secreted by goblet cells. Endotoxin, a bacterial product, has recently been reported to inhibit enterocyte migration in vitro through an increase of $\alpha 3 \beta 1$ integrin expression in intestinal epithelial cells (35). We show here that MFG-E8 produced by lamina propria macrophages plays an important role in regulating enterocyte migration along the cryptvillus axis. Our findings, together with previous investigations, suggest that the "trialogue" among lumenal contents, epithelial lining, and cells in lamina propria directs the enterocyte crypt-villus migration, which is required for the maintenance of intestinal barrier homeostasis and integrity.

It is unclear how MFG-E8 preserves intestinal epithelial homeostasis. Previous investigations show that MFG-E8 is required for clearance of apoptotic cells by macrophages (9). Recent studies suggest that MFG-E8 affects vascular/endothelial tissues and mammary gland homeostasis through mediating engulfment of apoptotic cells by macrophages $(36,37)$. In the present study, we show that MFG-E8 deficiency results in delayed epithelial cell renewal and turnover. However, in a pilot study, we found that MFG-E8-knockout mice had no increase in dying cells in the small intestinal crypts (Wang et al., unpublished observations). This indicates that a defect in MFG-E8 expression does not influence the removal of apoptotic epithelial cells in the gut. Together our results suggest that MFG-E8 regulates the homeostasis in intestinal lining by modulating the mobility of intestinal epithelial cells rather than by facilitating the clearance of apoptotic cells by macrophages. Therefore, it is probable that the molecular mechanisms through which MFG-E8 functions in vivo vary, depending on the tissue type. 


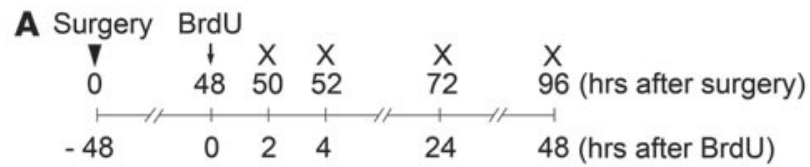

B

Time after BrdU injection (h)
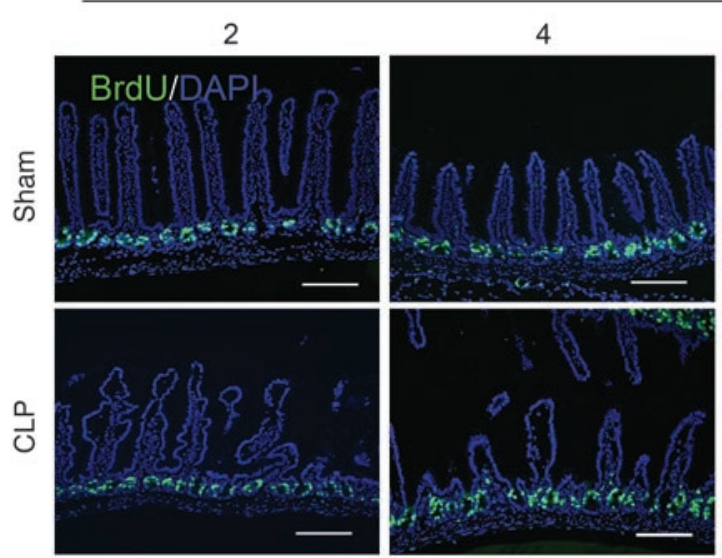

24
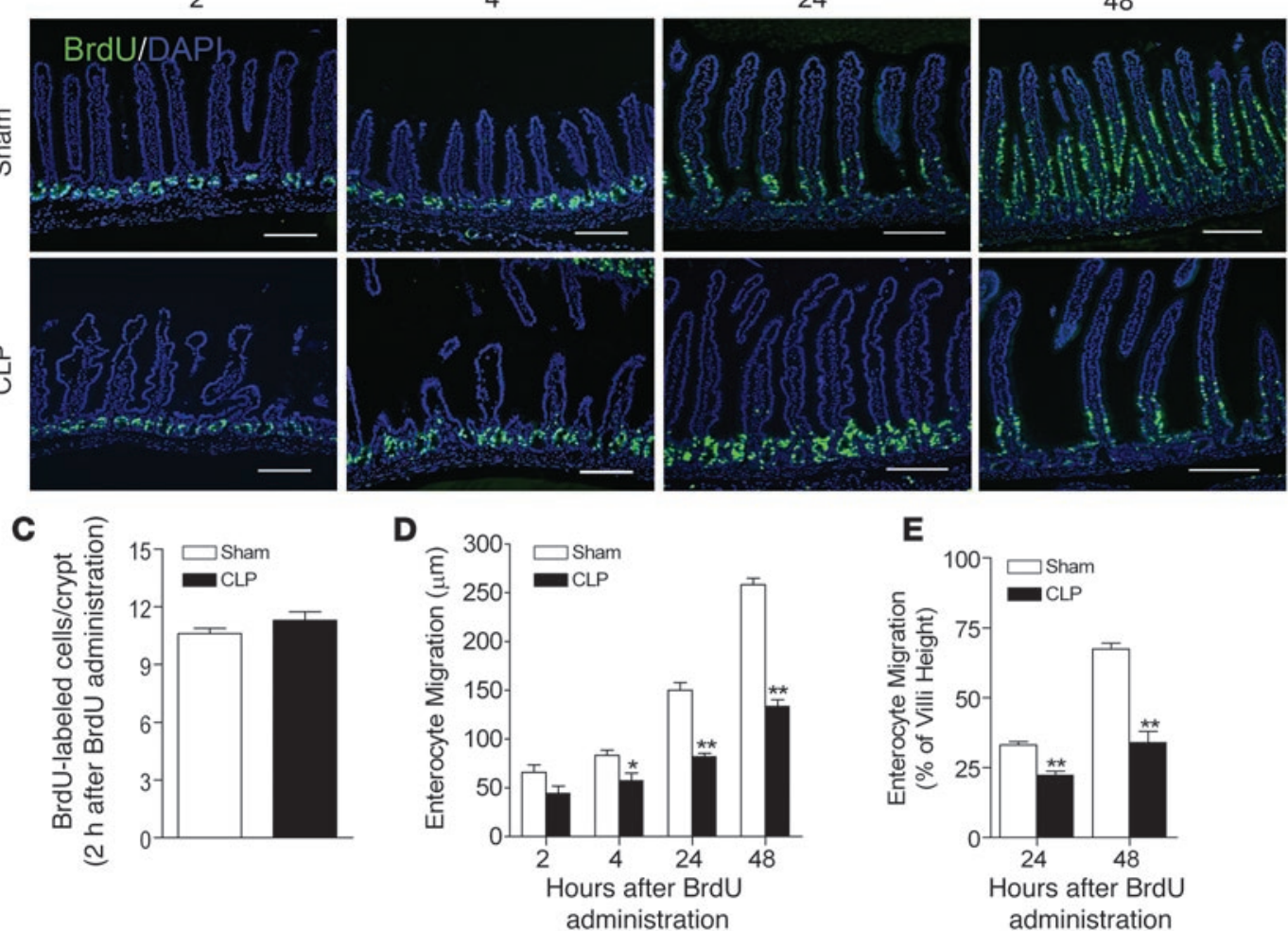

Figure 8

Enterocyte crypt-villus migration is slowed in septic mice. (A) Schedule for surgery, BrdU injection, and animal sacrifice. Mice were subjected to surgery (arrowhead), either CLP or sham operation, injection of BrdU (50 mg/kg BrdU; arrow), and euthanasia (X) at various time points as indicated. Small intestinal tissues were removed at the time of sacrifice and processed for routine histology. Tissue sections were examined after staining with anti-BrdU antibody. Nuclei were counterstained with DAPI. BrdU-labeled enterocyte migration was analyzed as described in Methods. (B) Typical photographs (merged profiles) of the small intestine are shown. BrdU-labeled cells are green; nuclei are blue. Scale bars: $150 \mathrm{um}$. (C) BrdU-labeled mitotic cells in crypts of the small intestine were quantitatively analyzed with immunostaining 2 hours after BrdU injection and immunostaining. $n=7$. ( $\mathbf{D}$ and $\mathbf{E})$ Quantitative analysis revealed a decrease in BrdU-labeled enterocyte crypt-villus migration after CLP. $n=7 .{ }^{* *} P<0.01$ versus sham group. ${ }^{*} P<0.05$.

Another major finding of the present study is that MFG-E8 plays an important role in the healing of damaged intestinal mucosa. Acute intestinal injury and mucosal barrier failure are frequent complications of critical conditions. Under pathophysiological conditions, maintenance and reestablishment of intact intestinal lining are thought to require both restitution and repair of damaged enterocytes by progenies of the intestinal stem cells from crypts. Previous investigations have shown that restitution is enhanced by various factors such as TGF- $\beta$, EGF, and ITF (5). We demonstrate that MFG-E8 promotes intestinal epithelial wound healing in vitro, and we provide data to support a role of MFG-E8 in epithelial restitution in vivo.

Little is known about how enterocyte crypt-villus migration is influenced by acute inflammatory insults and which factors are involved in regulating this event in sepsis. The data presented in this study demonstrate that, in sepsis, the upward crypt-to-vil- lus movement of enterocytes is inhibited. Furthermore, we have shown that intestinal MFG-E8 expression is down-regulated, which correlates with the defect of enterocyte migration and development of intestinal injury. In contrast, ITF expression is increased in the small intestine after sepsis challenge. Many restitution factors such as TGF- $\beta$ and PGE2 have also been shown to be increased in inflammation, indicating that downregulation of MFG-E8 appears to be a unique event associated with development of intestinal injury. In addition, we have identified that MFG-E8 binds to intestinal epithelium in vivo. Remarkably, we restored enterocyte migration along the crypt-villus axis in septic mice with administration of MFG-E8. Furthermore, we show that mucosal healing is delayed in MFG-E8-null mice. These observations suggest the importance of MFG-E8 in the maintenance and repair of mucosal barrier in inflammatory conditions. To our knowledge, this is the first report showing an association 
A

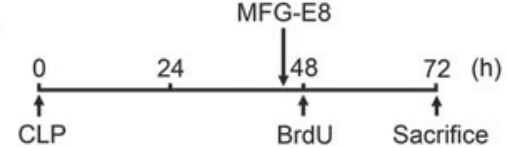

B $24 \mathrm{~h}$ after BrdU injection C
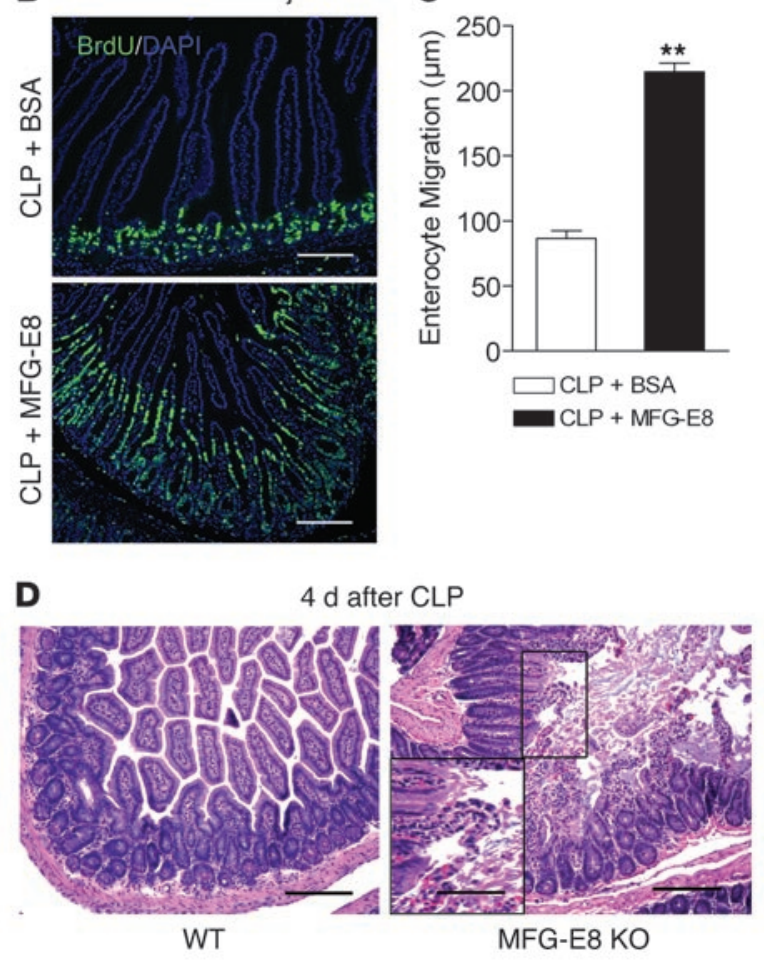

between MFG-E8 expression and defective enterocyte migration/restitution in sepsis. Our findings support the hypothesis that decreased MFG-E8 deters mucosal wound healing in sepsis. Recombinant MFG-E8 may have great potential in the prevention and treatment of intestinal injury in patients with severe systemic inflammatory response.

\section{Methods}

Animals. C57BL/6 wild-type mice (male, 6-9 weeks old) were purchased from The Jackson Laboratory. MFG-E8/SED1-knockout mice were produced as previously described (10). They were backcrossed to C57BL/6 for 10 generations, so they were considered to be fully congenic with the C57BL/6 background. Wild-type C57BL/6 mice were used as controls. Animals were housed in a specific pathogen-free animal facility at the Children's Memorial Research Center. All animal experiments were conducted in accordance with NIH guidelines and were approved by the Institutional Animal Care and Use Committee of the Children's Memorial Hospital.

Cell culture. IEC-18 cells (a nontransformed rat small intestinal epithelial cell line, passages 10-18 after receipt from American Type Culture Collection) were cultured in a water-saturated atmosphere with $5 \% \mathrm{CO}_{2}$ at $37^{\circ} \mathrm{C}$ in Dulbecco's modified Eagle's minimum essential medium supplemented with $5 \%$ heat-inactivated fetal bovine serum, insulin $(0.1 \mathrm{U} / \mathrm{ml}), 1 \%$ nonessential amino acids, $100 \mathrm{U} / \mathrm{ml}$ penicillin, and $100 \mu \mathrm{g} / \mathrm{ml}$ streptomycin.

Histology and general protocols for immunofluorescence. All animals were sacrificed by carbon dioxide inhalation. Intestinal tissues were immediately harvested and fixed in $10 \%$ buffered formalin overnight and processed for routine histology.

\section{Figure 9}

MFG-E8 is essential for maintenance of intestinal mucosal homeostasis and integrity in septic mice. (A-C) Treatment with recombinant MFG-E8 restores enterocyte crypt-villus migration in septic mice. (A) Experimental protocol. MFG-E8 (2 mg/kg, i.p.) and BrdU (50 mg/kg, i.p.) were given to mice at the times indicated. After sacrificing the animals, small intestinal tissues were processed for immunostaining. (B) MFG-E8 restored intestinal epithelial cell migration along cryptto-villus axis in septic mice. Scale bars: $150 \mu \mathrm{m}$. (C) Increase in BrdU-labeled enterocyte crypt-villus migration after treatment with MFG-E8, as revealed by quantitative analysis. $n=7$. ${ }^{* *} P<0.01$ versus CLP + BSA group. (D) Endogenous MFG-E8 is required for intestinal repair after septic challenge. Wild-type and MFG-E8-knockout mice were subjected to sublethal CLP and sacrificed 4 days later. Small intestinal tissues were processed for routine histology. H\&E stain. The inset shows mucosal injury in a selected area at higher magnification. Scale bars: $150 \mu \mathrm{m} ; 75 \mu \mathrm{m}$ (inset).

For detection of MFG-E8 and F4/80 in tissues, paraffin sections $(5 \mu \mathrm{m})$ were stained with hamster $\mathrm{mAb}$ to murine MFG-E8 (1:250; MBL Inc.) and biotin-labeled rat $\mathrm{mAb}$ to murine F4/80 (5 $\mu \mathrm{g} / \mathrm{ml}$; Serotec Ltd.). A goat anti-hamster IgG antibody labeled with Alexa Fluor 546 (1:250; Molecular Probes) and a streptavidin-labeled Alexa Fluor 633 (1:250; Molecular Probes) were used to detect MFG-E8 and F4/80 antibodies, respectively. Nuclei were counterstained with DAPI (Vector Lab). Slides were examined under an upright fluorescence microscope (model MD R; Leica Microsystems) using appropriate filters. Images were acquired with a digital camera (model C4742-95; Hamamatsu), transferred to a G4 Macintosh computer (Apple Computer), analyzed by the image analysis software Openlab, and assembled with Adobe Photoshop 8.0 software.

We also determined MFG-E8 bound to the cell surface with immunofluorescence. Cells were fixed with 3.7\% paraformaldehyde (Electron Microscopy Sciences) and then stained for MFG-E8 as described above.

For detection of F-actin cytoskeleton and Arp2/3, IEC-18 cells were fixed in $3.7 \%$ paraformaldehyde for 10 minutes, permeabilized with $0.05 \%$ Triton X-100 for 5 minutes, and blocked for 30 minutes in $10 \%$ goat serum. Rabbit anti-p34-Arc/ARPC2 antibody (1:100; Upstate Biotechnology) was incubated for 1 hour and amplified with Cy3-conjugated antirabbit IgG (1:400). F-actin was stained with FITC-conjugated phalloidin $(1 \mu \mathrm{g} / \mathrm{ml}$; Sigma-Aldrich). Nuclei were counterstained with DAPI. Cells were examined under a fluorescence microscope. The image was analyzed as described above. Under wound-healing conditions, the migrating cells at the migrating front were classified into 2 phenotypes, type I and type II, by the F-actin cytoskeleton structure (see Results for definitions). At least 500 cells were analyzed in each calculation of the 2 phenotypes.

Wound-healing model for cell migration assay. Confluent IEC-18 cells were maintained in serum-free medium for 24 hours before being processed for the assay. The cell monolayers were scrapped with a single-edge razor blade and washed with DMEM. Then the wounded monolayers were incubated with serum-free medium containing appropriate compounds for 5 hours at $37^{\circ} \mathrm{C}$ followed by examination under an inverted microscope (Model DM-IRB; Leica Microsystems). Images were acquired with a digital camera and transferred to a G4 Macintosh computer (Apple Computer). Migration was assessed in a blind fashion by measuring with the image analysis software Openlab and calculating as the total denuded area covered by migrated cells per unit width of wound.

RNA silencing. A standard protocol for RNA silencing was used (38). The RNAiFect reagent (Qiagen) was used for introducing siRNA into cells. siRNA target sequences described by Li et al. (15) were used: siPKC $\varepsilon$, 5'-AAGACGACCGATCCAAGTCAG-3' (bases 1212-1232, GenBank acces- 
sion no. NM017171); and control siRNA, 5'-AAGTACGTTGCGTCTACGTAG-3'. They were purchased from Qiagen.

In situ binding assay. Four hours after wounding, IEC-18 cell monolayers were treated with binding buffer $(140 \mathrm{mM} \mathrm{NaCl}, 2.5 \mathrm{mM} \mathrm{CaCl} 2,10 \mathrm{nM}$ HEPES, pH 7.4) containing MFG-E8 $(0.5 \mu \mathrm{g} / \mathrm{ml})$ or/and FITC-annexin V ( $5 \mu \mathrm{l} / 100 \mu \mathrm{l}$ binding buffer; Invitrogen) for 15 minutes at room temperature, washed with binding buffer, fixed in $3.7 \%$ paraformaldehyde for 10 minutes, processed for immunofluorescent staining with $\mathrm{mAb}$ to MFG-E8, and examined under a fluorescent microscope as described above. In some experiments, cells were pre-incubated with binding buffer containing annexin $\mathrm{V}$ $(100 \mu \mathrm{g} / \mathrm{ml})$ for 15 minutes before proceeding with the binding assay.

Measurement of enterocyte migration in vivo with the BrdU labeling strategy. Mice were subjected to i.p. injection of BrdU ( $50 \mathrm{mg} / \mathrm{kg}$; BD Biosciences). At the time points designated, mice were sacrificed by carbon dioxide inhalation. The entire small intestine was removed, flushed with normal saline, fixed with $10 \%$ buffered formalin, and processed for paraffin embedding and sectioning. Deparaffinized tissue sections $(5 \mu \mathrm{m})$ were boiled in citric acid buffer (0.01 mM, pH 6.0) for 20 minutes and slowly cooled down at room temperature for 40 minutes for antigen retrieval. They were then stained with rat anti-BrdU antibody (1:100; Accurate Chemical). Alexa Fluor 488 goat antibody against rat IgG (1: 250; Invitrogen) was used to detect antiBrdU antibody. Nuclei were counterstained with DAPI. Slides were examined under an upright fluorescence microscope using appropriate filters. The image was analyzed using OpenLab software as described above. The distance of enterocyte migration was the gap between the crypt base and the highest labeled cell within the crypt-villus axis. The villus height was also measured. Twenty crypts and villi per mouse were assessed and the mean migration distance and height values were used in the subsequent analysis. Preliminary experiments showed that sublethal CLP induced focal villous necrosis in mouse ileum (Supplemental Figure 5). In CLP mice, we only selected intact villi in order to examine how enterocytes migrated along the crypt-villus axis.

CLP. The procedure was modified from our previously described protocol (18). Briefly, mice were anesthetized by Nembutal (55 mg/kg, i.p.). After laparotomy, the distal portion of the cecum $(1 \mathrm{~cm})$ was ligated with 5-0 silk suture. The ligated cecum was then punctured with a 25 -gauge needle and slightly compressed with an applicator until a small amount of stool appeared. In sham-operated animals, the cecum was manipulated but without ligation and puncture, and was placed back in the peritoneum. The incision was closed using a 2-layer procedure: 5-0 silk suture on the muscle layer and the skin respectively. Mice received $1 \mathrm{ml}$ of saline i.p. for fluid resuscitation at the time of closure and $1 \mathrm{mg} / \mathrm{kg}$ buprenex s.c. 4 times at 12-hour intervals to minimize distress.

Neutralization of endogenous MFG-E8 with anti-MFG-E8 antibody. Normal and CLP-challenged mice were treated i.p. with a hamster monoclonal antibody against murine MFG-E8 (2 mg/kg; MLB Inc.). Control animals were injected with an equal amount of hamster isotype control IgG (eBioscience Inc.). Animals were sacrificed at appropriate time points.

Treatment of CLP mice with recombinant MFG-E8. CLP-challenged mice were injected i.p. with recombinant murine MFG-E8 (2 mg/kg; R\&D Systems). The control animals were injected with PBS buffer containing $50 \mu \mathrm{g} / \mathrm{ml}$ BSA (i.e., carrier protein). They were sacrificed at appropriate time points.

Western blotting. We used our standard protocol for isolation of total protein from intestinal tissues and immunoblotting (18). Rabbit polyclonal antibody against murine MFG-E8 (1:500; Santa Cruz Biotechnology Inc.), goat polyclonal antibody against murine ITF (1:500; Santa Cruz Biotechnology Inc.), and rat $\mathrm{mAb}$ to murine F4/80 (1:500; Serotec Ltd.) were used to detect MFG-E8, ITF, and F4/80 respectively.

Statistics. Data are expressed as means \pm SEM. ANOVA followed by Fisher's least significant difference post-hoc test were used to assess the significance of differences. $P<0.05$ was considered significant.

\section{Acknowledgments}

This work was supported in part by grants R01DK064240 (to X.-D. Tan) and R01HD23479 (to B.D. Shur) from the NIH and the Eloise and Warren Batts Investigator Chair (to X.-D. Tan). X.-L. Zuo is a visiting scholar partially supported by the Department of Gastroenterology, Qilu Hospital, Medical College of Shandong University, Jinan, Shandong Province, People's Republic of China. We thank Nicholas M. Lamm for technical assistance. Additionally, we thank Marianne E. Reed for preparation of the manuscript.

Received for publication February 15, 2007, and accepted in revised form September 13, 2007.

Address correspondence to: Xiao-Di Tan, Molecular and Cellular Pathobiology Program, Children's Memorial Research Center, Children's Memorial Hospital, 2300 Children's Plaza, Box 217, Chicago, Illinois 60614, USA. Phone: (773) 755-6380; Fax: (773) 755-6581; E-mail: xtan@northwestern.edu.

Heng-Fu Bu and Xiu-Li Zuo contributed equally to this work.
1. Radtke, F., Clevers, H., and Riccio, O. 2006. From gut homeostasis to cancer. Curr. Mol. Med. 6:275-289.

2. Yang, Q., Bermingham, N.A., Finegold, M.J., and Zoghbi, H.Y. 2001. Requirement of Math1 for secretory cell lineage commitment in the mouse intestine. Science. 294:2155-2158.

3. Hall, P.A., Coates, P.J., Ansari, B., and Hopwood, D. 1994. Regulation of cell number in the mammalian gastrointestinal tract: the importance of apoptosis. J. Cell Sci. 107:3569-3577.

4. van Es, J.H., et al. 2005. Wnt signalling induces maturation of Paneth cells in intestinal crypts. Nat. Cell Biol. 7:381-386.

5. Blikslager, A.T., Moeser, A.J., Gookin, J.L., Jones, S.L., and Odle, J. 2007. Restoration of barrier function in injured intestinal mucosa. Physiol. Rev. 87:545-564.

6. Stubbs, J.D., et al. 1990. cDNA cloning of a mouse mammary epithelial cell surface protein reveals the existence of epidermal growth factor-like domains linked to factor VIII-like sequences. Proc. Natl. Acad. Sci. U. S. A. 87:8417-8421.

7. Yolken, R.H., et al. 1992. Human milk mucin inhib- its rotavirus replication and prevents experimental gastroenteritis. J. Clin. Invest. 90:1984-1991.

8. Thery, C., et al. 1999. Molecular characterization of dendritic cell-derived exosomes. Selective accumulation of the heat shock protein hsc73. J. Cell Biol. 147:599-610.

9. Hanayama, R., et al. 2002. Identification of a factor that links apoptotic cells to phagocytes. Nature. 417:182-187.

10. Ensslin, M.A., and Shur, B.D. 2003. Identification of mouse sperm SED1, a bimotif EGF repeat and discoidin-domain protein involved in sperm-egg binding. Cell. 114:405-417.

11. Taylor, M.R., Couto, J.R., Scallan, C.D., Ceriani, R.L., and Peterson, J.A. 1997. Lactadherin (formerly BA46), a membrane-associated glycoprotein expressed in human milk and breast carcinomas, promotes Arg-Gly-Asp (RGD)-dependent cell adhesion. DNA Cell Biol. 16:861-869.

12. Andersen, M.H., Berglund, L., Rasmussen, J.T., and Petersen, T.E. 1997. Bovine PAS-6/7 binds alpha $v$ beta 5 integrins and anionic phospholipids through two domains. Biochemistry. 36:5441-5446.
13. Silvestre, J.S., et al. 2005. Lactadherin promotes VEGF-dependent neovascularization. Nat. Med. 11:499-506.

14. Oshima, K., et al. 1999. Lactation-dependent expression of an mRNA splice variant with an exon for a multiply O-glycosylated domain of mouse milk fat globule glycoprotein MFG-E8. Biochem. Biophys. Res. Commun. 254:522-528.

15. Li, Y., Urban, J.M., Cayer, M.L., Plummer, H.K., 3rd, and Heckman, C.A. 2006. Actin-based features negatively regulated by protein kinase C-epsilon. Am.J. Physiol. Cell Physiol. 291:C1002-C1013.

16. Nusrat, A., Delp, C., and Madara, J.L. 1992. Intestinal epithelial restitution. Characterization of a cell culture model and mapping of cytoskeletal elements in migrating cells. J. Clin. Invest. 89:1501-1511.

17. Lotz, M.M., Rabinovitz, I., and Mercurio, A.M. 2000. Intestinal restitution: progression of actin cytoskeleton rearrangements and integrin function in a model of epithelial wound healing. Am. J. Pathol. 156:985-996.

18. Bu, H.F., et al. 2006. Lysozyme-modified probiotic 
components protect rats against polymicrobial sepsis: role of macrophages and cathelicidin-related innate immunity. J. Immunol. 177:8767-8776.

19. Dignass, A., Lynch-Devaney, K., Kindon, H., Thim, L., and Podolsky, D.K. 1994. Trefoil peptides promote epithelial migration through a transforming growth factor beta-independent pathway. J. Clin. Invest. 94:376-383.

20. Tan, X.D., Chen, Y., Liu, Q., Gonzalez-Crussi, F., and Liu, X. 2000. Prostanoids mediate the protective effect of trefoil factor 3 in oxidant-induced intestinal epithelial cell injury: role of cyclooxygenase-2. J. Cell Sci. 113:2149-2155.

21. Chen, Y.H., Lu, Y., De Plaen, I.G., Wang, L.Y., and Tan, X.D. 2000. Transcription factor NF-kappaB signals antianoikic function of trefoil factor 3 on intestinal epithelial cells. Biochem. Biophys. Res. Commun. 274:576-582.

22. Martin, S.J., et al. 1995. Early redistribution of plasma membrane phosphatidylserine is a general feature of apoptosis regardless of the initiating stimulus: inhibition by overexpression of $\mathrm{Bcl}-2$ and $\mathrm{Abl}$. J. Exp. Med. 182:1545-1556.

23. Frasch, S.C., et al. 2000. Regulation of phospholipid scramblase activity during apoptosis and cell activation by protein kinase Cdelta. J. Biol. Chem. 275:23065-23073.

24. van den Eijnde, S.M., et al. 2001. Transient expression of phosphatidylserine at cell-cell contact areas is required for myotube formation. J. Cell Sci. 114:3631-3642.

25. Frasch, S.C., et al. 2004. Phospholipid flip-flop and phospholipid scramblase 1 (PLSCR1) co-localize to uropod rafts in formylated Met-Leu-Phe-stimulated neutrophils. J. Biol. Chem. 279:17625-17633.

26. Newton, A.C. 1995. Protein kinase C: structure, function, and regulation. J. Biol. Chem. 270:28495-28498.

27. Finkielstein, C.V., Overduin, M., and Capelluto, D.G. 2006. Cell migration and signaling specificity is determined by the phosphatidylserine recognition motif of Rac1. J. Biol. Chem. 281:27317-27326.

28. Ensslin, M.A., and Shur, B.D. 2007. The EGF repeat and discoidin domain protein, SED1/MFG-E8, is required for mammary gland branching morphogenesis. Proc. Natl. Acad. Sci. 104:2715-2720.

29. McKaig, B.C., Makh, S.S., Hawkey, C.J., Podolsky, D.K., and Mahida, Y.R. 1999. Normal human colonic subepithelial myofibroblasts enhance epithelial migration (restitution) via TGF-beta3. Am. J. Physiol. 276:G1087-G1093.

30. Ridley, A.J., Comoglio, P.M., and Hall, A. 1995. Regulation of scatter factor/hepatocyte growth factor responses by Ras, Rac, and Rho in MDCK cells. Mol. Cell Biol. 15:1110-1122.

31. Uberall, F., et al. 1997. Conventional PKC-alpha, novel PKC-epsilon and PKC-theta, but not atypical PKC-lambda are MARCKS kinases in intact NIH
3T3 fibroblasts. J. Biol. Chem. 272:4072-4078.

32. Song, J.C., Hrnjez, B.J., Farokhzad, O.C., and Matthews, J.B. 1999. PKC-epsilon regulates basolateral endocytosis in human T84 intestinal epithelia: role of F-actin and MARCKS. Am. J. Physiol. 277:C1239-C1249

33. Wong, M.H., Rubinfeld, B., and Gordon, J.I. 1998 Effects of forced expression of an NH2-terminal truncated beta-Catenin on mouse intestinal epithelial homeostasis. J. Cell Biol. 141:765-777.

34. Mashimo, H., Wu, D.C., Podolsky, D.K., and Fishman, M.C. 1996. Impaired defense of intestinal mucosa in mice lacking intestinal trefoil factor. Science. 274:262-265.

35. Qureshi, F.G., et al. 2005. Increased expression and function of integrins in enterocytes by endotoxin impairs epithelial restitution. Gastroenterology. 128:1012-1022.

36. Ait-Oufella, H., et al. 2007. Lactadherin deficiency leads to apoptotic cell accumulation and accelerated atherosclerosis in mice. Circulation. 115:2168-2177.

37. Atabai, K., et al. 2005. Mfge 8 is critical for mammary gland remodeling during involution. Mol. Biol. Cell. 16:5528-5537.

38. Zhu, Y.Q., and Tan, X.D. 2005. TFF3 modulates NF-kappaB and a novel negative regulatory molecule of NF-kappaB in intestinal epithelial cells via a mechanism distinct from TNF-alpha. Am. J. Physiol. Cell Physiol. 289:C1085-C1093. 